\title{
Remarks on Cosmological Spacetimes and Constant Mean Curvature Surfaces
}

\author{
Robert Bartnik \\ Centre for Mathematical Analysis, Australian National University, GPO Box 4, \\ Canberra ACT 2601, Australia
}

\begin{abstract}
We give a general condition which ensures the existence of constant mean curvature (CMC) Cauchy surfaces in cosmological spacetimes. However, there is an example of a spacetime which does not satisfy this condition and does not admit any CMC Cauchy surfaces. We discuss conditions under which $\mathrm{CMC}$ surfaces may exist.
\end{abstract}

\section{Introduction}

A Lorentzian manifold $\mathscr{V}$ is a cosmological spacetime if it is globally hyperbolic with compact Cauchy surfaces and satisfies the timelike convergence condition

$$
\operatorname{Ric}(T, T) \geqq 0 \text {, for every timelike vector } T \text {. }
$$

In this paper we are concerned with the following conjecture:

Conjecture 1. There is a CMC Cauchy surface in $\mathscr{V}$.

We first show the following existence result:

Theorem. Suppose the condition

$$
\mathscr{V}-I(p) \text { is compact, for some point } p \in \mathscr{V},
$$

is satisfied. Then there is a regular CMC Cauchy surface, which passes through $p$.

By regular surface we mean a strictly spacelike $C^{2, \alpha}$ hypersurface [B2] and we are assuming throughout that the spacetime metric is $C^{2}$. The proof of the theorem depends on some new existence and regularity results for prescribed mean curvature surfaces [B2].

However, Conjecture 1 is false in general, as we have:

Example 1. There is a cosmological spacetime $\mathscr{V}$ which admits no CMC Cauchy surfaces. 
In fact, $\mathscr{V}$ has no Cauchy surface with mean curvature everywhere nonnegative (or everywhere non-positive), so it is also an example of a cosmological spacetime with singularities which are not crushing [ES]. However, $\mathscr{V}$ does admit non-compact CMC surfaces (without boundary). We give two proofs of the nonexistence of CMC Cauchy surfaces, one based on the topology of the Cauchy surface (similar to the motivating example of Brill [BD]), and a second using conjugate points. In the discussion (Sect. 3) we consider this indicated relationship between the spatial and causal topologies.

The main result towards the conjecture is that of Gerhardt [GC], who showed the existence of CMC surfaces for cosmological spacetimes admitting past and future barriers [GC, B1]; i.e. regular Cauchy surfaces $S_{0}, S_{1}$ with $S_{1} \subset I^{+}\left(S_{0}\right)$ and mean curvatures $H_{0}, H_{1}$ respectively, satisfying

$$
\min _{S_{0}} H_{0} \geqq \max _{S_{1}} H_{1}
$$

The existence of such barriers is an easy consequence of our theorem, so it follows that $(\mathrm{G})$ is a stronger condition than (1). This is shown explicitly by Example 2 in the discussion section. Condition $(\mathrm{G})$ was first introduced by Geroch [GR] and later used by Galloway [GG1] (assuming $(\mathrm{G})$ held at every point of the spacetime) to prove a splitting theorem. In fact, the theorem yields a strengthening of Galloway's result:

Corollary 2. Suppose $(\mathrm{G})$ is satisfied at $p \in \mathscr{V}$, a cosmological spacetime. Then either $\mathscr{V}$ is a metric product or $\mathscr{V}$ is timelike incomplete.

The corollary is motivated by

Conjecture 2. Suppose $\mathscr{V}$ is a cosmological spacetime. Then either $\mathscr{V}$ is timelike geodesically incomplete or it splits as a metric product.

This is related to Yau's splitting conjecture [Y], recently proven by Eschenburg [E]: a timelike geodesically complete, globally hyperbolic spacetime satisfying TCC and admitting a line (i.e. a past and future unbounded maximising timelike geodesic) is a metric product. In fact, the assumption of timelike geodesic completeness can be removed [GG2]. It is not hard to show that $(\mathrm{G})$ implies the spacetime is either timelike geodesic incomplete or has a line, so Corollary 2 also follows from Eschenburg's result. We note that, in contrast to Corollary 2 and Conjecture 2, Yau's conjecture does not require the Cauchy surfaces be compact.

\section{Proof of the Theorem}

We first recall the Brill-Flaherty uniqueness result [BF]. If $M_{0}, M_{1}$ are two regular surfaces and $\gamma:[0, d] \rightarrow \mathscr{V}$ is a maximising future-timelike geodesic with $\gamma(0) \in M_{0}$ and $\gamma(d) \in M_{1}$, then

$$
0 \geqq \int_{0}^{d} \operatorname{Ric}\left(\gamma^{\prime}, \gamma^{\prime}\right) d s+H_{1}(\gamma(1))-H_{0}(\gamma(d))
$$


(Note that our conventions give the future unit hyperboloid in Minkowski space mean curvature $+n$.) Such a geodesic $\gamma$ occurs in many cases - for example if $M_{0}, M_{1}$ are compact Cauchy surfaces, or if $\partial M_{0}=\partial M_{1}$ and $M_{0}, M_{1}$ are compact $T$-homotopic surfaces [B2].

By [B2, Theorem 4.1], for any $\lambda \in \mathbb{R}$ there is a Cauchy surface $M_{\lambda}$ with $p \in M_{\lambda}$ and such that $M_{\lambda}-\{p\}$ is a regular hypersurface with $\mathrm{CMC} \lambda$. Although we shall not need it here, we note that $M_{\lambda}-\{p\}$ is either regular or tangent to the upper or lower light cone at $p$ [B3]. We see that the $M_{\lambda}$ are unique as follows: suppose $M, M^{*}$ are two regular surfaces with common (topological) boundary and with the same constant mean curvature. We may suppose $M^{*} C I^{+}(M)$, so the function

$$
d(x)=\sup \left\{d(x, y), y \in M^{*}\right\}
$$

[where $d(x, y)$ is the Lorentzian distance] is zero on $\partial M$ and has a positive maximum on $M$. By the implicit function theorem [CB], applied to a subdomain of $M$ with smooth boundary sufficiently close to $\partial M$, there is a CMC surface $M^{\prime}$ close to $M$ with lesser mean curvature, such that $d(x), x \in M^{\prime}$, attains an interior maximum on $M^{\prime}$. The resulting maximal geodesic contradicts (2).

From the uniqueness it follows that the surfaces $M_{\lambda}, \lambda \in \mathbb{R}$, form a foliation in $\mathscr{V}-J(p)$. [In fact, we can show this foliation covers all of $\mathscr{V}-J(p)$, but this isn't needed here.] Let $S$ be any regular Cauchy surface with $p \in S$ and define

$$
\Lambda=1+\sup _{S}\left|H^{0}\right|,
$$

where $H^{0}$ is the mean curvature of $S$. Note it also follows from (2) that $M_{A} \subset J^{-}(S)$ and dually, $M_{-1} \subset J^{+}(S)$.

Now fix a coordinate system $(x, t)$ centred at $p$ such that the metric $g_{\alpha \beta}(x, t)$ in the $(x, t)$ coordinates satisfies

$$
\begin{aligned}
\left|g_{\alpha \beta}-\eta_{\alpha \beta}\right|(x, t) & \leqq C\|(x, t)\|^{2}, \\
\left|\partial_{\gamma} g_{\alpha \beta}\right|(x, t) & \leqq C\|(x, t)\|, \\
\left|\partial_{\delta} \partial_{\gamma} g_{\alpha \beta}\right|(x, t) & \leqq C, \quad 0 \leqq \alpha, \beta, \gamma, \delta \leqq n,
\end{aligned}
$$

for a constant $C$, where $\eta_{\alpha \beta}$ is the flat Minkowski metric and $\|(x, t)\|^{2}=|x|^{2}+t^{2}$. By [B2, Proposition 5.1 and Theorem 5.2], there is a cylinder neighbourhood

$$
\mathscr{U}=\mathscr{U}(\varepsilon)=\{(x, t):|x| \leqq \varepsilon,|t| \leqq 2 \varepsilon\}
$$

for some $\varepsilon>0$ such that if $M$ is any regular hypersurface with constant mean curvature $H_{M}$ satisfying $\left|H_{M}\right| \leqq \Lambda$ and $\partial(M \cap \mathscr{U}) \subset\{(x, t):|x|=\varepsilon\}$, then $M \cap \mathscr{U}$ is the unique hypersurface with its boundary and mean curvature, in its $T$-homotopy class. For any $\lambda \in \mathbb{R}$, we denote by $D_{\lambda}=\operatorname{graph} u_{\lambda}$ the regular hypersurface in $\mathscr{U}$ solving the Dirichlet problem

$$
H_{M}=\lambda, \quad \partial M=M_{\lambda} \cap\{(x, t):|x|=\varepsilon\} .
$$

Since the $M_{\lambda}$ form a foliation, the boundaries $\partial D_{\lambda}, \lambda \in \mathbb{R}$, form a continuous 1-parameter family of $C^{2, \alpha}$ submanifolds. The uniqueness of the $D_{\lambda}$ and the regularity estimates for prescribed mean curvature surfaces with smooth boundary [B1, Sect. 3] imply the $D_{\lambda},-\Lambda \leqq \lambda \leqq \Lambda$, form a continuous family of regular 
hypersurfaces. Since $M_{A} \subset J^{-}(S)$, from the maximum principle we see that $D_{\Lambda} \subset I^{-}(S)$ and in particular, $u_{\Lambda}(0)<0$. Similarly, $u_{-\Lambda}(0)>0$ and by continuity there is $\lambda^{*} \in(-\Lambda ; \Lambda)$ such that $p \in D_{\lambda^{*}}$. We claim $D_{\lambda^{*}}=M_{\lambda^{*}} \cap \mathscr{U}$, and thus $M_{\lambda^{*}}$ is a regular CMC Cauchy surface. To see this construct a foliation $Q_{\lambda},-\Lambda \leqq \lambda \leqq \Lambda$, with mean curvature $\lambda^{*}$ and boundary values $\partial\left(M_{\lambda} \cap \mathscr{U}\right)$. The existence of such a foliation follows from [B2, Sect. 5]. Now $D_{\lambda^{*}}=Q_{\lambda^{*}}$, so $\partial\left(\left(M_{\lambda^{*}}-\{p\}\right) \cap \mathscr{U}\right) \subset D_{\lambda^{*}}$, and the claim follows from the maximum principle [or (2)], applied to $\left(M_{\lambda^{*}}-\{p\}\right) \cap \mathscr{U}$ and $Q_{\lambda}$, $\lambda>\lambda^{*}$ and $\lambda<\lambda^{*}$. q.e.d.

\section{Example 1}

The construction is an adaption of an idea of Brill [BD] and starts with the Tolman-Bondi metric

$$
d s^{2}=-d t^{2}+X^{2} d r^{2}+Y^{2} d \Omega^{2},
$$

where $d \Omega^{2}$ is the standard metric on $S^{2}$ and $X=X(r, t), Y=Y(r, t)$. With pressure free perfect fluid matter (i.e. dust) and assuming the marginally unbound condition $Y^{\prime}=X\left({ }^{\prime}=\partial / \partial r\right)$, the Einstein equations can be integrated [ES], giving

$$
\begin{aligned}
X(r, t) & =\left(M^{\prime}(r)\left(t_{0}(r)-t\right)+2 M(r) t_{0}^{\prime}(r)\right) /\left(6 M(r)^{2}\left(t_{0}(r)-t\right)\right)^{1 / 3}, \\
Y(r, t) & =\left(9 / 2 M(r)\left(t_{0}(r)-t\right)^{2}\right)^{1 / 3}
\end{aligned}
$$

for some functions $M(r), t_{0}(r)$. Two examples of this metric are:

(i) Schwarzschild spacetime,

$$
M(r)=1, t_{0}(r)=r \quad \text { for } \quad-\infty<r<\infty, t<r,
$$

where the $(r, t)$ coordinates cover one half the maximally extended Schwarzschild solution, and

(ii) Friedman universe with $k=0$,

$$
M(r)=r^{3}, t_{0}(r)=0 \quad \text { for } \quad 0 \leqq r, t<0 .
$$

The Oppenheimer-Snyder stellar model [OS] is obtained by attaching these two solutions along $r=1$, with $r \leqq 1$ the Friedman solution and $r>1$ the Schwarzschild solution. We do the opposite: define

$$
\begin{aligned}
t_{0}(r)=r, M(r)=1 & \text { for } \quad-\infty<r \leqq 1-\varepsilon \text { (Schwarzschild region), } \\
t_{0}(r)=1, M(r)=r^{3} & \text { for } \quad 1+\varepsilon \leqq r \text { (Friedman region), }
\end{aligned}
$$

where $\varepsilon>0$ is small and for $1-\varepsilon \leqq r \leqq 1+\varepsilon$ we choose $M(r), t_{0}(r)$ smoothly so that

$$
\begin{gathered}
t_{0}^{\prime}(r) \geqq 0, t_{0}^{\prime \prime}(r) \leqq 0, M^{\prime}(r) \geqq 0, M^{\prime \prime}(r) \geqq 0, \\
t_{0}^{\prime}(r)^{2}+M^{\prime}(r)^{2}>0 .
\end{gathered}
$$

Then $X(r, t)>0$ so the $(r, t)$ coordinates are non-singular (no "shell-crossing" singularities) and the matter density

$$
4 \pi \varrho(r, t)=M^{\prime}(r) X^{-1} Y^{-2} \geqq 0,
$$

so the resulting spacetime satisfies the timelike convergence condition. 


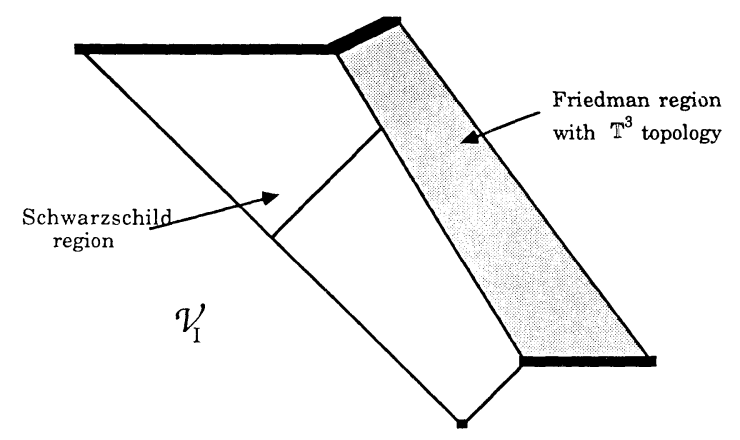

In the Friedman region the metric is

$$
-d t^{2}+(9 / 2)^{2 / 3}(1-t)^{4 / 3}\left(d r^{2}+r^{2} d \Omega^{2}\right)
$$

Changing to Euclidean spatial coordinates $(x, y, z)$ we see that spatial translations are isometries and hence the faces of the cube $\max (|x|,|y|,|z|)=2$ can be identified, giving a $C^{\infty}$ induced metric on the resulting spacetime $\mathscr{V}_{\text {I }}$ (see Fig. 1). Brill's spacetime is constructed from $\mathscr{V}_{\text {I }}$ by attaching the other half of the maximally extended Schwarzschild solution to the boundary of the Schwarzschild component of $\mathscr{V}_{\mathrm{I}}$, giving an asymptotically flat spacetime with Cauchy surfaces topologically $\mathbb{T}^{3}-\{p t\}$.

Instead we attach $\mathscr{V}_{\text {I }}$ to a time-inverted copy $\mathscr{V}_{\text {II }}$ of itself, again along the boundary of the Schwarzschild region (Fig. 2). The resulting spacetime $\mathscr{V}$ is clearly globally hyperbolic with compact Cauchy surfaces diffeomorphic to $\mathbb{T}^{3} \# \mathbb{T}^{3}$ and having two disjoint Friedman regions separated by a piece of the interior of the maximally extended Schwarzschild solution.

Proposition. $\mathscr{V}$ has no CMC Cauchy surfaces. However, there is $K_{0}>0$ such that for any constant $K$ with $|K| \geqq K_{0}$, there is a noncompact boundaryless regular surface with constant mean curvature $K$ and diffeomorphic to $\mathbb{T}^{3}-\{p t$.$\} .$

Proof. Observe that $\mathscr{V}$ has a time-inverting isometry $\varphi: \mathscr{V} \rightarrow \mathscr{V}$ which interchanges the two components $\mathscr{V}_{\mathrm{I}}, \mathscr{V}_{\mathrm{II}}$. Suppose $S$ is a regular CMC Cauchy surface and let $S^{*}=\varphi(S)$. Then $H\left(S^{*}\right)=-H(S)$ and we may assume $H(S)>0$ [the case

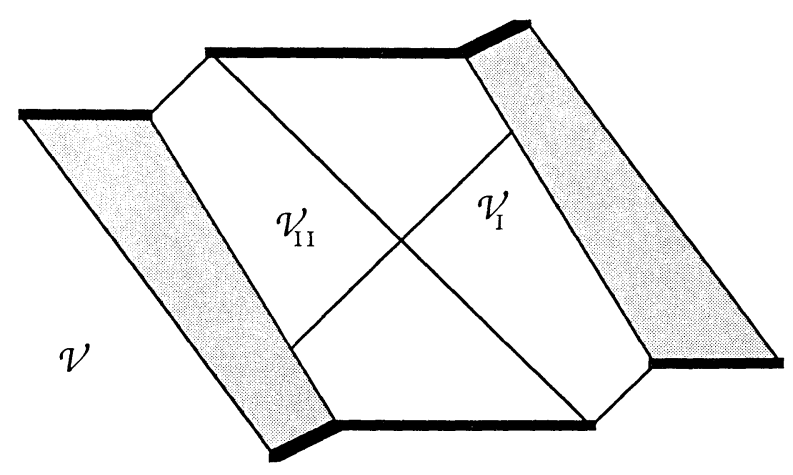


Fig. 3

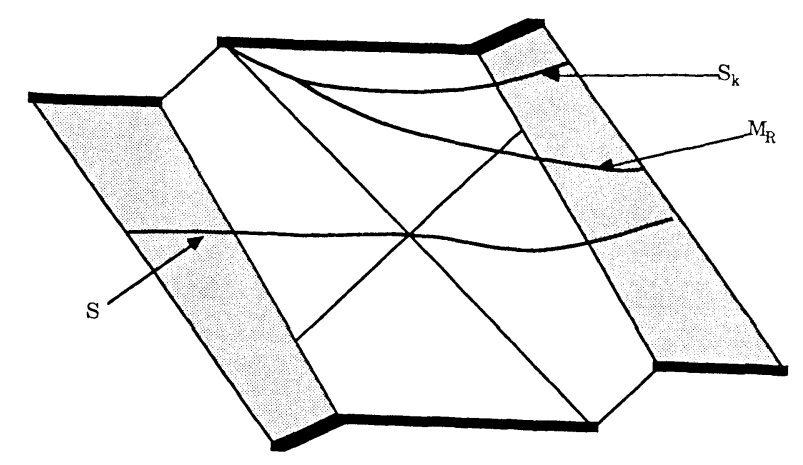

$H(S)=0$ will be dealt with later], so by $(2), S^{*} \subset I^{+}(S)$. Thus $S, S^{*}$ are past, future barrier surfaces respectively for a maximal surface and by [B1, GC] there is a maximal Cauchy surface $S^{* *}$ in $I^{+}(S) \cap I^{-}\left(S^{*}\right)$. But $\pi_{1}\left(S^{* *}\right)=\pi_{1}\left(\mathbb{T}^{3} \# \mathbb{T}^{3}\right)=\mathbb{Z}^{3} * \mathbb{Z}^{3}$ $>\mathbb{Z}^{2}=\pi_{1}\left(\mathbb{T}^{2}\right)$, so by $[\mathrm{SY}] S^{* *}$ cannot admit a metric of non-negative scalar curvature. But $\mathscr{V}$ satisfies the weak energy condition, so any maximal surface has non-negative scalar curvature. This is the contradiction.

To show the existence of non-compact CMC surfaces, we first note that the surfaces $S_{k}$ in $\mathscr{V}_{\text {I }}$ defined by

$$
t=t_{0}(r)-k, \quad 0<k \leqq k_{0},
$$

have mean curvature $\leqq-(2 k)^{-1}$ for some $k_{0}>0$. This follows from a straightforward but tedious computation and estimation using (9) (see also [ES]) and is made plausible by noting that in the separate Schwarzschild and Friedman regions the surfaces $S_{k}$ are the well-known crushing surfaces. Note that $S_{k} \approx \mathbb{T}^{3}-\{p t$. $\}$ with the metric near the point that of $S^{2} \times \mathbb{R}$.

Now let $S$ be any fixed regular Cauchy surface in $\mathscr{V}$. Choose $k \leqq k_{0}, R \leqq 0$ and $-(2 k)^{-1}<K<\inf _{S} H(S)$ and consider the Dirichlet problem

$$
\begin{gathered}
\text { find a regular surface } M_{R} \text { such that } H\left(M_{R}\right)=K, \\
\partial M_{R}=\partial\left(S_{k} \cap\{r \geqq R\}\right) \text { and } M_{R} \text { is } \partial_{t} \text {-homotopic to } S_{k} \cap\{r \geqq R\}
\end{gathered}
$$

(see Fig. 3). By [B2] this has a regular solution in the precompact region $I^{-}\left(S_{k} \cap\{r \geqq R\}\right) \cap I^{+}(S)$, since $S_{k}$ is a future barrier surface and $S$ is a past barrier. Since $M_{R^{*}} \cap I^{-}\left(S_{k} \cap\{r \geqq R\}\right) \cap I^{+}(S) \neq \emptyset$ for all $R, R^{*} \leqq 0$, the sequence $\left\{M_{R}\right\}_{R \leqq 0}$ has a limiting surface $M$. From [B2, Theorem 3.8], $M$ is a regular surface with mean curvature $K$ and $\partial_{t}$-homotopic to $S_{k}$, provided we can show there are no endless null geodesics contained within $M$. This follows from the global hyperbolicity of $\mathscr{V}$ and the fact that $M_{R} \subset I^{+}(S)$ for all $R \leqq 0$. q.e.d.

There is an interesting alternative argument that the example $\mathscr{V}$ does not admit a CMC Cauchy surface, which does not rely on the topology of the surface, and only uses TCC:

Suppose $S$ is such a surface, with non-zero mean curvature, which we may assume negative. By the focal point lemma [HE], there is a finite upper bound to the length of a future timelike curve from $S$. However, there are future inextendible 
timelike curves in $\mathscr{V}$ which follow the boundary of the dust cloud (see Fig. 3) and have infinite length, which is a contradiction. To show there are no maximal Cauchy surfaces, we can use the following:

Lemma. Suppose $S$ is a maximal Cauchy surface in a cosmological spacetime. Then there is a neighbourhood of $S$ foliated by CMC Cauchy surfaces.

Proof. Let $\mathfrak{M}(u)$ denote the mean curvature of $\operatorname{graph}_{S}(u), u \in C^{\infty}(S)$, in Gaussian normal coordinates. Now $\mathfrak{M}$ has linearisation

$$
\mathfrak{M}^{\prime}(0)=-\Delta_{S}+|A|^{2}+\operatorname{Ric}(N, N),
$$

where $A($,$) is the second fundamental form of S$ and $\operatorname{Ric}(N, N)$ is the Ricci tensor in the direction normal to $S$. Clearly $\mathfrak{M}^{\prime}(0)$ is invertible and there are non-zero CMC surfaces near $S$ unless $|A|^{2} \equiv 0$ and $\operatorname{Ric}(N, N) \equiv 0[\mathrm{CB}]$. In this case we introduce the operator

$$
\mathfrak{M}^{*}: C^{\infty}(S) \times \mathbb{R} \rightarrow C^{\infty}(S) \times \mathbb{R}, \mathfrak{M}^{*}(u, k)=\left(\mathfrak{M}(u)-k, \int_{S} u\right),
$$

which has invertible linearisation about $(0,0)$ since the kernel of $\mathfrak{M}^{\prime}(0)$ contains only the constant functions. By the inverse function theorem, for all $\varepsilon$ sufficiently small there is $u(\varepsilon)$ such that $\mathfrak{M}(u(\varepsilon))=k=k(\varepsilon)$ and $\int_{S} u(\varepsilon)=\varepsilon$. Since $u^{\prime}(0) \in \operatorname{ker} \mathfrak{M}^{\prime}(0)$, this implies $u^{\prime}(0)=$ const $>0$ and thus the graphs of the $u(\varepsilon)$ form a foliation in a neighbourhood of $S$ by CMC surfaces. q.e.d.

If the foliation is by maximal surfaces, then the regularity results imply the foliation extends, and the metric must be a product. Since $\mathscr{V}$ is clearly not static, this shows there can be no CMC Cauchy surfaces in $\mathscr{V}$. q.e.d.

We note the lemma has the immediate

Corollary 1. Suppose $\mathscr{V}$ is a cosmological spacetime with a regular CMC Cauchy surface. Then either $\mathscr{V}$ is timelike geodesically incomplete or $\mathscr{V}$ splits globally as a metric product (cf. [GG1, GC]).

Combining this with the CMC existence theorem gives the splitting result, Corollary 2, mentioned in the introduction. The focal point argument can be modified (see below) to show Brill's original example cannot have any Cauchy surfaces with non-positive mean curvature (and in particular, has no maximal Cauchy surface).

Example 1 illustrates the difficulty in applying Eschenburg's result to prove Conjecture 2: although there is a sequence of pairs of points $\left(p_{n}, q_{n}\right)$ tending to $\left(l^{-}\right.$, $\left.i^{+}\right)$with $d\left(p_{n}, q_{n}\right) \rightarrow \infty$, the limit of the connecting timelike geodesics is null, so there is no line.

\section{Discussion}

Now let us consider what these results tell us about the general existence question for $\mathrm{CMC}$ surfaces. Firstly, notice that the two arguments exploit apparently quite 
distinct properties of $\mathscr{V}$, the first relying on the topology of the Cauchy surfaces, whilst the second uses the global causal structure.

We will say that a hypersurface has bad topology if its fundamental group contains a surface group (so the hypersurface does not admit a positive scalar curvature metric). The first argument shows that a cosmological spacetime which also satisfies the weak energy condition (this does not follow from TCC) and whose Cauchy surface has bad topology, cannot have positive and negative mean curvature barriers unless the resulting maximal surface is a totally geodesic $\mathbb{T}^{3}$ with vanishing energy density along the hypersurface. A simple example shows that this exceptional case can occur:

Example 2. Consider the globally hyperbolic spacetime $\mathscr{W}$ with coordinates $\left\{(x, t) \in \mathbb{R}^{3,1}:|t|<1\right\}$ and conformal metric $g=\Omega^{2}(t) \eta$, where $\eta$ is the standard Minkowski metric and $\Omega(t)=\exp \left(-t^{2} /\left(1-t^{2}\right)\right)$ for $|t|<1$.

It is easy to check that the timelike convergence condition is strictly satisfied for any unit timelike vector $N$, we have $\operatorname{Ric}(N, N) \geqq 6$ - and the weak energy condition is also satisfied, $T(N, N) \geqq 0$, with equality exactly for the normal vectors to the Cauchy surface $\{(x, t): t=0\}$. This surface is totally geodesic by symmetry, and by identifying the sides of a spatial cube of side length $L$, we obtain a family of cosmological spacetimes $\mathscr{W}_{L}$ with maximal Cauchy surfaces having bad topology.

This example has some other interesting properties. Firstly, the dominant energy condition is not satisfied and cannot be satisfied by any non-trivial choice of $\Omega(t)$. Secondly, although TCC is satisfied strictly and WE holds almost everywhere, a generic local perturbation about $\{t=0\}$ of the metric $g$ cannot satisfy WE. This follows from the fact that a generic metric does not admit totally geodesic hypersurfaces. By the barrier argument, the perturbed spacetime will still admit a (unique) maximal Cauchy surface, with scalar curvature $R=2 T(N, N)$ $+|A|^{2}$. Since $|A| \neq 0$ and $\mathbb{T}^{3}$ does not admit a metric of non-negative scalar curvature, the weak energy condition must fail somewhere along the maximal surface.

Finally we note that for $L>1$, the spacetimes $\mathscr{W}_{L}$ do not satisfy $(\mathrm{G})$ but do have past and future mean curvature barriers, so $(G)$ is strictly stronger than Gerhardt's barrier condition.

The second argument relies on the existence of unbounded timelike curves and, as indicated above, can be adapted to non-compact Cauchy surfaces by assuming additionally that the (future) unbounded curve $\gamma$ satisfies

$$
J^{-}(\gamma) \cap S \text { compact }
$$

where $S$ is any Cauchy surface. Note again that Brill's example satisfies this condition. The argument then is as follows. As before, there can be no negative mean curvature Cauchy surface. Furthermore, if there were a maximal Cauchy surface $S$, then by solving the Dirichlet problem for negative mean curvature and boundary $\partial\left(J^{-}(\gamma) \cap S\right)$, we would again contradict the focal point lemma.

We can paraphrase (11) by saying there is a hidden (future) infinity. It seems reasonable to conjecture that 
Conjecture 3. An asymptotically flat, globally hyperbolic spacetime satisfying TCC has a maximal Cauchy surface if and only if there are no hidden infinities.

The only if part is shown above. The most convincing evidence for the converse is the "interior condition" in the existence result of [B1], which postulates a uniform bound for the "height" of the domain of dependence of the intersection of any Cauchy surface and the "interior region." This condition immediately precludes the existence of any hidden infinity.

In [SY2] it is shown that an asymptotically flat spacetime satisfying the dominant energy condition and with Cauchy surfaces having bad topology must necessarily have an apparent horizon. Together with Brill's example, this suggests there may be a relation between the existence of hidden infinities and bad topology. In any case, we could also assume the dominant energy condition and add "iff the Cauchy surfaces have good topology" to the conjecture.

With (11) modified to " $J^{-}(\gamma) \cap S$ compactly contained in $S$," there is a similar conjecture for the existence of CMC Cauchy surfaces in cosmological spacetimes. Although we have seen that $(G)$ is not a necessary condition in this case, if we also require the spacetime to have past and/or future unbounded timelike curves, then there is a close connection between the failure of $(\mathrm{G})$ and the existence of hidden infinities (and hence the non-existence of any CMC Cauchy surfaces).

\section{References}

[B1] Bartnik, R.: Existence of maximal surfaces in asymptotically flat spacetimes. Commun. Math. Phys. 94, 155-175 (1984)

[B2] Bartnik, R.: Regularity of variational maximal surfaces. CMA report R22-86, to appear in Acta Math.

[B3] Bartnik, R.: Isolated singular points of Lorentz mean curvature surfaces. CMA report R 39-86

[BD] Brill, D.: On spacetimes without maximal surfaces. In: Proc. Third Marcel Grossman meeting, Ning, H. (ed.). Amsterdam: North-Holland 1982

[BF] Brill, D., Flaherty, F.: Isolated maximal surfaces in spacetime. Commun. Math. Phys. 50, 157-165 (1976)

[CB] Choquet-Bruhat, Y.: Spacelike submanifolds with constant mean extrinsic curvature of a Lorentzian manifold. Ann. Sci. Norm. Super. Pisa 3, 361-376 (1976)

[ES] Eardley, D., Smarr, L.: Time functions in numerical relativity: marginally bound dust collapse. Phys. Rev. D 19, 2239-2259 (1979)

[E] Eschenburg, J.-H.: The splitting theorem for space-times with strong energy condition (preprint)

[GG1] Galloway, G.: Splitting theorems for spatially closed spacetimes. Commun. Math. Phys. 96, 423-429 (1984)

[GG2] Galloway, G.: Private communication

[GC] Gerhardt, C.: $H$-surfaces in Lorentzian manifolds. Commun. Math. Phys. 89, 523-553 (1983)

[GR] Geroch, R.: Singularities in closed universes. Phys. Rev. Lett. 17, $445-447$ (1966)

[H] Hawking, S.: Singularities in the universe. Phys. Rev. Lett. 17, $444-445$ (1966)

[OS] Oppenheimer, J., Snyder, H.: On continued gravitational contraction. Phys. Rev. 56, 455-459 (1939) 
[SY 1] Schoen, R., Yau, S.-T.: Existence of incompressible minimal surfaces and the topology of 3-dimensional manifolds with non-negative scalar curvature. Ann. Math. 110, 127-142 (1979)

[SY2] Schoen, R., Yau, S.-T.: Proof of the positive mass conjecture. II. Commun. Math. Phys. 79, 231-260 (1981)

[Y] Yau, S.-T.: Problems in differential geometry. In: Seminar on Differential Geometry. Yau, S.-T. (ed.). Ann. Math. Studies 102

Communicated by S.-T. Yau

Received November 6, 1986; in revised form October 1, 1987 